MCGILL- $93 / 46$

sw 9404

\title{
PHOTOPRODUCTION OF LARGE- $p_{T}$ HADRONS BY POLARIZED BEAM AND TARGET
}

\author{
J.J. Peralta, ${ }^{a}$ A.P. Contogouris, ${ }^{a, b}$ B. Kamal ${ }^{a}$ and F. Lebessis ${ }^{c}$
}

\begin{abstract}
a. Department of Physics, McGill University, Montreal H3A 2T8, Canada
b. Nuclear and Particle Physics, University of Athens, Athens 15771, Greece

c. Institute of Nuclear Physics, NRCPS Democritos, Athens 15310, Greece
\end{abstract}

\begin{abstract}
Production at large transverse momentum $\left(p_{T}\right)$ of hadrons by a polarized photon beam on a longitudinally polarized proton target is studied. The contributions due to the structure of the photon are taken into account and discussed. In certain kinematic domains cross sections and event rates sizeable enough for successful experiments are predicted and single hadron production is shown to be a good probe of the size of the polarized gluon distribution. A particularly clean probe is found to be a special combination of cross sections for production of two large- $p_{T}$ hadrons. The feasibility of high energy polarized photon beams is also discussed.
\end{abstract}




\section{Introduction}

So far the numerous tests of perturbative QCD (PQCD), which have led us to believe that it is a very successful theory of strong interactions, are experiments on reactions involving unpolarized partons and particles. One of these tests - quite successful - has been photoproduction of hadrons at large $p_{T} \cdot{ }^{(1)(2)}$ One may call the tests with unpolarized reactions first generation tests.

The EMC experiment on the longitudinally polarized structure function of the proton $^{(3)}$ marks the beginning of a second generation of tests, perhaps more stringent than of the first. The results of this experiment are further enforced by the recent measurement of the spin-dependent structure function of the deuteron. ${ }^{(4)}$ Several other experiments with polarized particles have been completed ${ }^{(5)}$ or proposed at HERA ${ }^{(6)}, \mathrm{RHIC}^{(6)(7)}$ or Fermilab. ${ }^{(8)}$ One may expect that large $p_{T}$ photoproduction of hadrons with polarized beam and target will eventually occupy a place.

The result of the EMC experiment was quite surprising: The valence quarks carry almost no spin of the proton! Within PQCD a solution was found in suggesting a large and positive polarized gluon distribution $\Delta g$ (together with small polarized sea). An alternative solution consists of a rather large polarized strange sea together with a not so large $\Delta g$.

In this work we study the PQCD predictions for large- $p_{T}$ photoproduction of hadrons by a polarized beam and proton target. In particular we present results with two sets of polarized parton distributions differing essentially in the size of $\Delta g$.

Section 2 presents the contributions to single hadron photoproduction of the main subprocesses. Sect. 3 presents the contributions due to the structure of the photon. Sect. 4 has our results on $\vec{\gamma} \vec{p} \rightarrow h+X$. Sect. 5 discusses twohadron photoproduction and shows that a proper combination of cross sections for 
$\vec{\gamma} \vec{p} \rightarrow h_{1}+h_{2}+X$ is, in principle, a clean probe of the size of $\Delta g$. Sect. 6 contains an overall assessment of the present work and our conclusions.

\section{Single hadron photoproduction._ Direct subprocesses.}

We begin with photoproduction of a single hadron, namely $\vec{\gamma}+\vec{p} \rightarrow h+X$. In an important part of the kinematic range, this is known to be dominated by the subprocesses $\vec{g} \vec{\gamma} \rightarrow q \bar{q}$ and $\vec{q} \vec{\gamma} \rightarrow q g$ in which $\gamma$ directly participates in the hard scattering process; of course, the coupling of the photon is point-like. Let $\vec{a}\left(p_{1}\right)+\vec{\gamma}\left(p_{2}\right) \rightarrow c\left(p_{3}\right)+d$ denote either of these (the quantities in parentheses are 4-momenta); we define:

$$
\hat{s}=\left(p_{1}+p_{2}\right)^{2} \quad \hat{t}=\left(p_{3}-p_{1}\right)^{2} \quad \hat{u}=\left(p_{3}-p_{2}\right)^{2}
$$

Next we write

$$
\Delta \frac{d \sigma}{d \hat{t}}=\frac{1}{2}\left\{\frac{d \sigma}{d \hat{t}}(a(+) \gamma(+) \rightarrow c d)-\frac{d \sigma}{d \hat{t}}(a(-) \gamma(+) \rightarrow c d)\right\}
$$

where \pm denote parton $a$ and photon helicities. For the inclusive cross section of $\vec{\gamma} \vec{p} \rightarrow h+X$ we write likewise:

$$
E \frac{\Delta d \sigma}{d^{3} p}=\frac{1}{2}\left\{E \frac{d \sigma}{d^{3} p}(p(+) \gamma(+) \rightarrow h X)-E \frac{d \sigma}{d^{3} p}(p(-) \gamma(+) \rightarrow h X)\right\}
$$

where \pm denote proton and photon helicities. Then the contribution of $\vec{a} \vec{\gamma} \rightarrow c d$ is:

$$
E \frac{\Delta d \sigma}{d^{3} p}\left(p_{T}, s, \eta\right)=\frac{1}{\pi} \sum_{q} e_{q}^{2} \int \frac{d x_{a}}{x_{a}} \frac{d z}{z^{2}} \Delta F_{a / p}\left(x_{a}, Q^{2}\right) D_{h / c}\left(z, Q^{2}\right) \Delta \frac{d \sigma}{d \hat{t}} \delta\left(1+\frac{\hat{t}+\hat{u}}{\hat{s}}\right)
$$

where $\Delta F_{a / p}$ the momentum distribution of a longitudinally polarized parton $a$ in the proton, $D_{h / c}$ the fragmentation function $c \rightarrow h$ and $e_{q}$ denotes quark charge. The subprocess cross sections are (see also Ref. 9):

$$
\Delta \frac{d \sigma_{g \gamma}}{d \hat{t}}=-\frac{\pi \alpha \alpha_{s}}{\hat{s}^{2}} \frac{\hat{t}^{2}+\hat{u}^{2}}{\hat{t} \hat{u}} \quad \Delta \frac{d \sigma_{q \gamma}}{d \hat{t}}=\frac{8 \pi \alpha \alpha_{s}}{3 \hat{s}^{2}} \frac{\hat{s}^{2}-\hat{t}^{2}}{-\hat{s} \hat{t}}
$$


where the outgoing antiquark (gluon) has momentum $p_{3}$. With $p_{T}$ the transverse momentum of $h$ and $\eta$ its c.m. rapidity with respect to the photon:

$$
z=\frac{1}{2} x_{T}\left(e^{\eta}+e^{-\eta} / x_{a}\right)
$$

where $x_{T}=2 p_{T} / \sqrt{s}(\sqrt{s}=$ c.m. energy $)$.

We are interested in photoproduction of charged hadrons $\left(h^{+}, h^{-}\right)$. These are mostly $\pi^{+}, \pi^{-}$, to a lesser extent $K^{+}, K^{-}$and even less protons; production of $\bar{p}$ and other baryons is negligible. For $\pi^{+}, \pi^{-}$we use the fragmentation functions of Ref. 10 and note the relations:

$$
D_{\pi^{+} / u}=D_{\pi-/ \bar{u}}=D_{\pi^{-} / d}=D_{\pi^{+} / \bar{d}} \quad D_{\pi^{-} / u}=D_{\pi^{+} / \bar{u}}=D_{\pi^{+} / d}=D_{\pi^{-/ \bar{d}}}
$$

and

$$
D_{\pi^{+} / g}=D_{\pi-/ g}
$$

Regarding fragmentation into $K^{ \pm}$we use the relations of Ref. 11

$$
D_{K^{+/ u}}=D_{K^{-/ \vec{u}}} \quad D_{K^{+/ \bar{s}}}=D_{K^{-/ s}} \quad \frac{D_{K^{-/ u}}}{D_{K^{+} / u}}=\frac{D_{\pi^{-} / u}}{D_{\pi^{+} / u}}
$$

and

$$
D_{K+/ s}=D_{K^{+/ d}}=D_{K+/ \bar{d}}=D_{K^{+} / \bar{u}}=D_{K^{-/ \bar{s}}}=D_{K^{-/ \bar{d}}}=D_{K-/ d}=D_{K-/ u}
$$

We take

$$
D_{K+/ u}=D_{K+/ \bar{s}}=\frac{1}{3} D_{\pi^{+} / u}
$$

we find that at $Q^{2} \simeq 4 \mathrm{GeV}^{2}$ the resulting $D_{K^{+} / u}\left(z, Q^{2}\right)$ and $D_{K^{+} / \bar{s}}\left(z, Q^{2}\right)$ are in fair agreement with the corresponding fragmentation functions of Ref. 11, as well as of Ref. 12. Finally we take $D_{K^{ \pm} / g}=0$ and neglect baryon production. In this way we find that photoproduction of all charged hadrons exceeds that of $\pi^{+}$and 
$\pi^{-}$by $\sim 40 \%$ in agreement with well known experimental facts on large $p_{T}$ hadron production. ${ }^{(13)}$

\section{Photon structure (resolved photon) contributions.}

In addition to the above direct subprocess, the photon may interact via two other mechanisms: $:^{(1)(2)}$

(i) Through its constituent $q \bar{q}$ pairs or gluons (partonic component); here, as in the direct subprocesses, the coupling of the photon is point-like.

(ii) By first turning into a vector meson state (hadronic component) one may consider that this state is eventually decomposed into constituents, which give rise to some hard scattering subprocess.

Both mechanisms (i) and (ii) correspond to so called resolved photon subprocesses. We begin with (i).

With definitions similar to (2.1)-(2.3) and considering $\gamma \rightarrow q \bar{q}$, the contributions from $\vec{a}\left(p_{1}\right)+\vec{q}\left(p_{2}\right) \rightarrow c\left(p_{3}\right)+d\left(p_{4}\right)$ to the inclusive cross section of $\vec{\gamma} \vec{p} \rightarrow h+X$ now has the form:

$$
\begin{gathered}
E \frac{\Delta d \sigma}{d^{3} p}=\frac{1}{\pi} \sum_{q} e_{q}^{2} \int \frac{d x_{a}}{x_{a}} \frac{d x_{b}}{x_{b}} \frac{d z}{z^{2}} \Delta F_{a / p}\left(x_{a}, Q^{2}\right) \Delta F_{q / \gamma}\left(x_{b}, Q^{2}\right) D_{h / c}\left(z, Q^{2}\right) \times \\
\times \frac{\Delta d \sigma}{d \hat{t}}(a q \rightarrow c d) \delta\left(1+\frac{\hat{t}+\hat{u}}{\hat{s}}\right)
\end{gathered}
$$

Here $\Delta F_{q / \gamma}\left(x, Q^{2}\right)$ is the polarized photon structure function for $\gamma \rightarrow q$. For unpolarized photons there have been extensive studies regarding the structure function $F_{q / \gamma}\left(\right.$ and $\left.F_{g / \gamma}\right) ;$ information on $\Delta F_{q / \gamma}\left(\right.$ and $\left.\Delta F_{g / \gamma}\right)$ is much more limited. For the sake of simplicity and convenience we will make use of the lowest nontrivial order of $\alpha_{s}$; or quark parton model (QPM) result: as we discuss below, the matter is eventually related with the effects of the hadronic component (mechanism (ii)). 
Thus we take:

$$
\Delta F_{q / \gamma}\left(x, Q^{2}\right)=\frac{\alpha}{2 \pi} \Delta P_{q / \gamma}(x) \log \frac{Q^{2}}{\tilde{\Lambda}^{2}}
$$

where the polarized split function ${ }^{(14)}$

$$
\Delta P_{q / \gamma}(x)=\frac{2 x-1}{2}
$$

and we take $\tilde{\Lambda}=\Lambda(=0.2 \mathrm{GeV})$; to this order:

$$
\Delta F_{g / \gamma}\left(x, Q^{2}\right) \equiv 0
$$

The subprocess cross sections $\frac{\Delta d \sigma}{d \hat{t}}(a q \rightarrow c d)$ have been determined in Ref. 9; with the notation

$$
\frac{\Delta d \sigma}{d \hat{t}}(a b \rightarrow c d)=\frac{\pi \alpha_{s}^{2}}{\hat{s}^{2}} \Delta \Sigma(a b \rightarrow c d)
$$

they are:

$$
\begin{gathered}
\Delta \Sigma(g q \rightarrow g q)=\frac{\hat{s}^{2}-\hat{t}^{2}}{\hat{u}^{2}}+\frac{4}{9} \frac{\hat{s}^{2}-\hat{t}^{2}}{-\hat{t} \hat{s}} \\
\Delta \Sigma\left(q_{\alpha} q_{\beta} \rightarrow q_{\gamma} q_{\delta}\right)=\frac{4}{9}\left(\frac{\hat{s}^{2}-\hat{t}^{2}}{\hat{u}^{2}}+\delta_{\alpha \beta}\left(\frac{\hat{s}^{2}-\hat{u}^{2}}{\hat{t}^{2}}-\frac{2}{3} \frac{\hat{s}^{2}}{\hat{t} \hat{u}}\right)\right) \\
\Delta \Sigma\left(q_{\alpha} \bar{q}_{\beta} \rightarrow q_{\gamma} \bar{q}_{\delta}\right)=\frac{4}{9}\left(\delta_{\alpha \gamma} \delta_{\beta \delta} \frac{\hat{s}^{2}-\hat{t}^{2}}{\hat{u}^{2}}-\delta_{\alpha \beta} \delta_{\gamma \delta} \frac{\hat{t}^{2}+\hat{u}^{2}}{3}-\frac{2}{3} \delta_{\alpha \gamma} \delta_{\beta \delta} \delta_{\gamma \delta} \frac{\hat{t}^{2}}{-\hat{u} \hat{s}}\right)
\end{gathered}
$$

At the low end of $p_{T}(\lesssim 2.5 \mathrm{GeV})$ the Fermi motion (intrinsic transverse momentum of the partons in the hadron) has some effect. However, in photoproduction this effect is much less important than in hadron-hadron reactions, and it will be neglected.

Now we turn to the discussion of $(3.2),(3.3)$ and begin by briefly recalling the situation in unpolarized $\gamma$ reactions; we follow Refs. 15.

In the QPM $F_{q / \gamma}$ has the same form as (3.2) with only (3.2a) replaced by $P_{q / \gamma}(x)$; this is its simplest form, in which regularization-dependent constant (i.e. only $x$-dependent) pieces are neglected. Correspondingly $F_{g / \gamma}\left(x, Q^{2}\right) \equiv 0$, as well. 
As for polarized $\gamma$, the result follows from the computation of a simple box graph. Inserted into the evolution equations, this contribution amounts to an inhomogeneous term. The full solution of these equations, to be termed leading order (LO) QCD, contains the homogeneous plus one particular inhomogeneous solution; the former is usually absorbed in the hadronic component. Then in the LO solution, $F_{q / \gamma}$ differs from that of QPM mainly near the kinematic endpoints $x=0$ and $x=1$. Of course, in LO, $F_{g / \gamma}\left(x, Q^{2}\right) \neq 0$.

In next-to-LO, for very large $Q^{2}$ (i.e. neglecting subdominant terms), $F_{q / \gamma}\left(x, Q^{2}\right)$ is given by the above inhomogeneous solution and is entirely calculable in QCD. This analysis was first made by Witten ${ }^{(16)}$ and led to the hope that the photon structure function could be fully predicted (i.e. including its $x$-dependence, apart from $\tilde{\Lambda}$ of Eq. (3.2)). Unfortunately the hope was shattered when it was realized that the singlet part of $F_{q / \gamma}$ contains a singularity at $x=0$. In higher order this singularity becomes worse, and so are the indications for even higher orders; near $x=0, F_{q / \gamma}$ even becomes negative! On the other hand, the full $F_{q / \gamma}$ is positive and regular; certain regularizing terms enter. ${ }^{(15)}$ The origin of them can be traced to the hadronic component (ii). ${ }^{(15)}$ Interesting ways to cancel the above singularities have been proposed, ${ }^{(17)}$ but the simplest (and perhaps safest) procedure seems the following: ${ }^{(18)}$ Accept $F_{q / \gamma}\left(x, Q_{0}^{2}\right)$ at some $Q_{0}^{2}=\mathcal{O}\left(1 \mathrm{GeV}^{2}\right)$ from experiment (input) and use QCD to determine the evolution. This is the procedure one follows for partonic distributions in hadrons.

Now we turn to $\Delta F_{q / \gamma}$ and $\Delta F_{g / \gamma}$. In LO these functions have been determined. ${ }^{(19)}$ First, regarding $\Delta F_{q / \gamma}$. Fig. 1 of Ref. 19 shows that, away from kinematic endpoints, they are much the same as Eqs. (3.2). (3.2a) in sign. magnitude and structure (notice in all cases a change of sign of $\Delta F_{q / \gamma}\left(x, Q^{2}\right)$ at some intermediate $x$ ). Thus, away from $x=0$ or 1 , we may reasonably expect similar results. ${ }^{(20)}$ Next. 
regarding $\Delta F_{g / \gamma}$, Fig. 2 of Ref. 19 shows that it is important at small $x$. Setting $\Delta F_{g / \gamma} \equiv 0$ (eq. (3.3)), at larger $x$ makes little difference.

In LO, at small $x$, all $\Delta F_{q / \gamma}, \Delta F_{g / \gamma}$ are $\sim x^{-1}$ (Ref. 19). For the next-to-LO nothing is known. Of course, no experimental input $\Delta F_{q / \gamma}\left(x, Q_{0}^{2}\right)$ is known, as well. Thus it appears to us that, away from $x=0$ or 1 , for simplicity's sake, use of (3.2) and (3.3) is not unjustified.

Furthermore, (3.2) and (3.3) are convenient to us, and this will be discussed in Sect. 5 .

To close this section we note that, in contrast to the subprocesses $\vec{g} \vec{\gamma} \rightarrow q \vec{q}$ and $\vec{q} \vec{\gamma} \rightarrow q g$ which lead to events with 3 -jet topology, the contributions due to the structure of the photon lead to 4 jets. The additional jet arises from the fragments of $\gamma$ and emerges in the photon's direction. Thus such contributions can be eliminated by excluding 4-jet events. Anyway we present predictions both with and without these contributions.

\section{Results on single hadron photoproduction.}

Subsequently we use the two sets of polarized parton distributions of Ref. 21 (set 1: large $\Delta g$, set 2: relatively small $\Delta g$ ) evolved with $Q^{2}$. In the running coupling $\alpha_{s}\left(Q^{2}\right)$ we take $\Lambda=0.2 \mathrm{GeV}$ and four flavours. Our results correspond to the scale $Q^{2}=p_{T}^{2}$, but we comment on other choices. We consider $\sqrt{s}=27 \mathrm{GeV}$ and $170 \mathrm{GeV}$, but these energies should be viewed as only indicative.

Fig. 1 shows the contributions to $\vec{\gamma} \vec{p} \rightarrow \pi^{ \pm}+X$ of the direct subprocesses, of the photon structure and of their sum for rapidities $\eta=1.5,0$ and -1.5 . The predictions correspond to set 1 of Ref. 21. In general the photon structure contributions are important at the lower $p_{T}$. The direct subprocesses dominate at $\eta=1.5$ and $\eta=0$. but not at $\eta=-1.5$. 
Fig. 2 shows the ratio of the inclusive cross sections for $\vec{\gamma} \vec{p} \rightarrow \pi^{ \pm}+X$ calculated with set 1 and set 2 . In particular at $\eta=1.5$ this ratio is near 2 and reflects the fact that at the indicated range of $p_{T}$ the subprocesses with initial gluon dominate and that in set $1 \Delta F_{g / p}$ is significantly larger than in set 2 . It appears that in this kinematic range $\vec{\gamma} \vec{p} \rightarrow \pi^{ \pm}+X$ is a good probe of the polarized gluon distribution.

Fig. 3 presents contributions of only the direct subprocesses to $\vec{\gamma} \vec{p} \rightarrow h^{ \pm}+X$, where $h$ denotes $\pi$ or $K$. Following the NA14 collaboration $^{(1)}$ we present inclusive cross sections integrated over bins of $p_{T}$, namely

$$
\Delta \frac{d^{2} \sigma}{d \eta d \phi}\left(p_{T}, s, \eta\right)=\int_{p_{T}-p_{0}}^{p_{T}+p_{0}} d p_{T}^{\prime} p_{T}^{\prime} E^{\prime} \frac{\Delta d \sigma}{d^{3} p^{\prime}}\left(p_{T}^{\prime}, s, \eta\right)
$$

For $\sqrt{s}=27 \mathrm{GeV}$ we take $p_{0}=0.5 \mathrm{GeV}$; for $\sqrt{s}=170: p_{0}=1 \mathrm{GeV}$. Again the predictions with set 1 exceed those of set 2 by factors $\sim 2$.

For the direct subprocess contributions we also determine the asymmetries

$$
A\left(p_{T}, s, \eta\right)=E \frac{\Delta d \sigma}{d^{3} p}\left(p_{T}, s, \eta\right) / E \frac{d \sigma}{d^{3} p}\left(p_{T}, s, \eta\right) ;
$$

these are presented in Fig. 4. To determine the unpolarized $E d \sigma / d^{3} p$ we use the unpolarized parton distributions of Ref. 22 (fit SL-leading order). In general $A$ are large and negative at the lower $p_{T}$. At fixed $s$, as $p_{T}$ increases $|A|$ decreases; this is due to the fact that, with increasing $p_{T}$, the subprocess $\vec{q} \vec{\gamma} \rightarrow q g$ becomes relatively more important; this subprocess contributes with sign opposite to that of $\vec{g} \vec{\gamma} \rightarrow q \bar{q}$ (cf. Eqs. (2.5)). At even larger $p_{T}$ (or $x_{T}$ ) $\vec{q} \vec{\gamma} \rightarrow q g$ dominates and $A$ changes sign. Again, at the lower $p_{T}$, where $\vec{g} \vec{\gamma} \rightarrow q \bar{q}$ dominates, $|A|$ for solution 1 exceeds that for solution 2 by factors $\sim 2$.

We have determined the event rates corresponding to our cross sections, and here we briefly state the main results. As a basis we take a differential luminosity of $15 \times 10^{30} \mathrm{~cm}^{-2} \mathrm{sec}^{-1}$ (to be achieved later at HERA) and $1 / 3$ of the year effective running time. 
Referring to solution 1 and Eq. (4.1), we denote by $N$ the per year number of events integrated over the bin $p_{T}-p_{0}<p_{T}^{\prime}<p_{T}+p_{0}$; we shall give the range of $N$ for $\eta$ varying in a range $\eta_{\max }>\eta>\eta_{\min }$. Then for solution $2, N$ can be trivially deduced from our Fig. 3.

So, for $\vec{\gamma} \vec{p} \rightarrow h^{ \pm}+X$, at $\sqrt{s}=27 \mathrm{GeV}$, for the bin $2<p_{T}^{\prime}<3 \mathrm{GeV}$ and for $1.5>\eta>-1$ we find $4 \times 10^{5} \gtrsim N \gtrsim 10^{4}$; in our kinematic range these correspond to the maximal rates. At $\sqrt{s}=170 \mathrm{GeV}$, for the bin $8<p_{T}^{\prime}<10 \mathrm{GeV}$ and for $1.5>\eta>-1.5: 7 \times 10^{3} \gtrsim N \gtrsim 20$; these correspond to the minimal rates. For $\gamma p \rightarrow h^{ \pm}+X$ (unpolarized) approximate rates can be deduced from Fig. 4 .

Leaving aside the technical difficulties in setting up polarized photon beams (see Sect. 6), it appears that, in general, our predicted cross sections and rates are sufficiently large for successful experiments.

Finally we have considered variations of the scale $Q^{2}$ in the usual range $p_{T}^{2} / 4 \leq$ $Q^{2} \leq 4 p_{T}^{2}$. Typically, for $Q^{2}=p_{T}^{2} / 4$ the polarized cross sections increase by factors of $2 \sim 2.5$ with respect to their value at $Q^{2}=p_{T}^{2}$; for $Q^{2}=4 p_{T}^{2}$, they decrease by factors $1.25 \sim 2$. In general, the asymmetries $A$ are less affected: For the whole range $p_{T}^{2} / 4 \leq Q^{2} \leq 4 p_{T}^{2} A$ changes by less than $20 \%$.

\section{Two-hadron photoproduction}

As we stated in the Introduction, the size of $\Delta g$ is a main issue in Spin Physics. Therefore it is of interest to isolate the subprocess $\vec{g} \vec{\gamma} \rightarrow q \bar{q}$. For this, as in Ref. 23, we consider the semi-inclusive production $\vec{\gamma} \vec{p} \rightarrow h_{1}+h_{2}+X$, where the hadrons $h_{i}, i=1,2$, are produced at large transverse momenta $k_{T i}$ in opposite azimuthal hemispheres.

To determine the contribution of the subprocess $\vec{a} \vec{\gamma} \rightarrow c d$. in the center of mass of the colliding $\gamma$ and $p$ take as $z$-axis the direction of $\gamma\left(\hat{z}=\hat{p}_{2}\right)$ and let $\theta_{i}$ and $\phi_{i}$ 
the polar and azimuthal angles defining the direction of $\vec{k}_{i}$ (Fig. 5). We can take $\phi_{1}=0$, so that the plane defined by $\vec{p}_{2}$ and $\vec{k}_{1}$ (scattering plane) coincides with the $x-z$ plane. Then, with $k_{i x}=k_{T i} \cos \phi_{i}$ and $\eta_{i}=\ell n \cot \frac{\theta_{i}}{2}$ the rapidity of $h_{i}$ one obtains

$$
\sigma\left(h_{1} h_{2}\right) \equiv \frac{\Delta d \sigma}{d \eta_{1} d^{2} k_{T 1} d \eta_{2} d k_{2 x}}=\frac{e_{q}^{2}}{\pi k_{T 1}} \Delta F_{a / p}(x) \frac{\Delta d \sigma}{d \hat{t}} D_{h_{1} / c}\left(z_{1}\right) D_{h_{2} / d}\left(z_{2}\right)
$$

where

$$
x=e^{-\eta_{1}-\eta_{2}}
$$

and

$$
z_{1}=\frac{k_{T 1}}{\sqrt{s}}\left(e^{\eta_{1}}+e^{\eta_{2}}\right) \quad z_{2}=\frac{k_{2 x}}{\sqrt{s}}\left(e^{\eta_{1}}+e^{\eta_{2}}\right) .
$$

In the above expression an integration over the component $k_{2 y}$ (perpendicular to the scattering plane) has been carried; also, in the expression of $\Delta d \sigma / d \hat{t}$ one has:

$$
\hat{s}=x s \quad \hat{t}=-\hat{s} e^{\eta_{1}} /\left(e^{\eta_{1}}+e^{\eta_{2}}\right) \quad \hat{u}=-s e^{-\eta_{1}} /\left(e^{\eta_{1}}+e^{\eta_{2}}\right)
$$

Now using the relations (2.7) and (2.8) for the fragmentation functions to $\pi^{+}$and $\pi^{-}$one can show that the combination of inclusive cross sections:

$$
\Delta(\pi) \equiv \sigma\left(\pi^{+} \pi^{-}\right)+\sigma\left(\pi^{-} \pi^{+}\right)-\sigma\left(\pi^{+} \pi^{+}\right)-\sigma\left(\pi^{-} \pi^{-}\right)
$$

isolates the contribution of the subprocess $\vec{\gamma} \vec{g} \rightarrow q \bar{q}$. One finds: ${ }^{(23)}$

$$
\Delta(\pi)=\frac{2\left(e_{u}^{2}+e_{d}^{2}\right)}{\pi k_{T 1}} \Delta F_{g / p}(x) \frac{\Delta d \sigma_{g \gamma}}{d \hat{t}}\left(D_{\pi^{+} / u}\left(z_{1}\right)-D_{\pi^{-} / u}\left(z_{1}\right)\right)\left(D_{\pi^{+} / u}\left(z_{2}\right)-D_{\pi^{-} / u}\left(z_{2}\right)\right)
$$

In a similar manner, using the relations (2.9) and (2.10) for the fragmentation functions to $K^{+}$and $K^{-}$and assuming simply $D_{K^{+/ g}}=D_{K^{-} / g}$, we find that the combination

$$
\Delta(K)=\sigma\left(K^{+} K^{-}\right)+\sigma\left(K^{-} K^{+}\right)-\sigma\left(K^{+} K^{+}\right)-\sigma\left(K^{-} K^{-}\right)
$$


isolates $\vec{\gamma} \vec{g} \rightarrow q \bar{q}$, as well. More specifically:

$$
\begin{gathered}
\Delta(K)=\frac{2}{\pi k_{T 1}} \Delta F_{g / p}(x) \\
\frac{\Delta d \sigma_{g \gamma}}{d \hat{t}} \sum_{q} e_{q}^{2}\left\{D_{K^{+/ q}}\left(z_{1}\right)\left(D_{K^{-} / \bar{q}}\left(z_{2}\right)-D_{K^{+} / \bar{q}}\left(z_{2}\right)\right)+\right. \\
\left.+D_{K^{-} / \bar{q}}\left(z_{1}\right)\left(D_{K^{-/ q}}\left(z_{2}\right)-D_{K^{+/ q}}\left(z_{2}\right)\right)\right\}
\end{gathered}
$$

where $q=u, d$ and $s$.

Regarding the contributions of the photon structure, working as in Sect. 3 to the lowest nontrivial order of $\alpha_{s}$ (QPM) it is not difficult to demonstrate that in the combinations $\Delta(\pi)$ and $\Delta(K)$ these contributions cancel. The reason is that $\Delta(\pi)$ and $\Delta(K)$ involve differences of oppositely charged pions or kaons.

Moreover, since the inclusive cross section $\sigma\left(h_{1} h_{2}\right)$ involves two hadrons in opposite hemispheres (Fig. 5) the effect of the Fermi motion is practically eliminated even at very low $k_{T i}$. Thus (5.6) and (5.8) are very clean probes of the polarized gluon distribution $\Delta F_{g / p}$.

Now we can see the convenience (in addition to simplicity) in using the QPM $\Delta F_{q / \gamma}$ (Eq. (3.2)): It facilitates the demonstration that in the combinations $\Delta(\pi)$ and $\Delta(K)$ the photon structure contributions cancel. For the LO $\Delta F_{q / \gamma}^{(19)}$, the cancellation is not complete. However, in view of the many similarities between QPM and LO distributions discussed in Sect. 3 and away from kinematic endpoints, we may reasonably expect cancellation to a significant degree.

Fig. 6 presents results for the quantity $\Delta(\pi)+\Delta(K)$. The corresponding inclusive cross sections are integrated over bins of $k_{T 1}$ and $k_{2 x}$, namely:

$$
\frac{\Delta d \sigma}{d \eta_{1} d \phi_{1} d \eta_{2}}\left(k_{T 1}, k_{2 x}, s, \eta_{1}, \eta_{2}\right)=\int_{k_{T 1}-k_{0}}^{k_{T 1}+k_{0}} k_{T 1}^{\prime} d k_{T 1}^{\prime} \int_{k_{2 x}-k_{0}}^{k_{2 x}+k_{0}} d k_{2 x}^{\prime} \frac{\Delta d \sigma}{d \eta_{1} d \eta_{2} d^{2} k_{T 1}^{\prime} d k_{2 x}^{\prime}}
$$

We consider $\eta_{1}=\eta_{2}=\eta$ and as before take for $\sqrt{s}=27 \mathrm{GeV}: k_{0}=0.5 \mathrm{GeV}$. for $\sqrt{s}=170$ at the larger $\eta$. the cross sections show a clear difference (by factors $\sim 2$ ) between solutions 1 and 2 . 
We have again determined event rates on the basis of the luminosity and running time of Sect. 4. Referring to solution 1 and Eq. (5.9), we denote by $N$ the per year number of events integrated over $k_{T 1}-k_{0}<k_{T 1}^{\prime}<k_{T 1}+k_{0}$; with $\eta=\eta_{1}=\eta_{2}$, for convenience, we give for $N=N(\eta)$ several charactéristic values (although just one would be sufficient, the others being determined from our Fig. 6).

At $\sqrt{s}=27 \mathrm{GeV}$, the bin of $k_{2 x}$ is $2.5<k_{2 x}^{\prime}<3.5 \mathrm{GeV}$ (Fig. 6); then for the bin $2<k_{T 1}^{\prime}<3$ we find: $N(\eta=1.5) \simeq 3.7 \times 10^{3}, N(\eta=1.25) \simeq 10^{4}\left(=N_{\max }\right)$ and $N(\eta=0.55) \simeq 423$. At $\sqrt{s}=170$ the bin of $k_{2 x}$ is $5<k_{2 x}^{\prime}<7$ (Fig. 6). Then for the bin $8<k_{T 1}^{\prime}<10$ we find: $N(\eta=1.5) \simeq 210, N(\eta=1) \simeq 19$ and $N(\eta=0.55) \simeq 0.7$.

Leaving aside practical difficulties (see Sect. 6), we may say that, at the larger $\eta$, our predicted rates are not discouraging.

Before closing this section we would like to explain qualitatively the shapes of $\Delta d^{3} \sigma / d \eta_{1} d \eta_{2} d \phi_{1}$ and their difference between $\sqrt{s}=27$ and $\sqrt{s}=170 \mathrm{GeV}$.

For $\sqrt{s}=170 \mathrm{GeV}$, with $\eta$ varying in the range $0.5<\eta<1.5, z_{i}$ are small (Eq. (5.3)) and the fragmentation functions $D_{h / q}\left(z_{i}\right)$ vary little, the behaviour of $\Delta(\pi)$ and $\Delta(K)$ is controlled by $\Delta F_{q / p}(x)$. Then as $\eta$ increases, $x$ decreases (Eq. $(5.2))$, and $\Delta F_{g / p}(x)$, which peaks at small $x$, increases. Hence $\left|\Delta d^{3} \sigma / d \eta_{1} d \eta_{2} d \phi_{1}\right|$ increases. Moreover, for the various bins of $k_{T 1}$, this cross section almost scales.

For $\sqrt{s}=27 \mathrm{GeV}, k_{T 1} / \sqrt{s}$ and $k_{2 x} / \sqrt{s}$ are not so small, and this complicates the shape. First, at fixed $k_{T 1}$ and $k_{2 x}$, beginning with $\eta=0.5$ and up to some value of $\eta$, the behaviour of $\Delta(\pi)$ and $\Delta(K)$ is again controlled by $\Delta F_{g / p}(x)$; thus, as for $\sqrt{s}=170,\left|\Delta d^{3} \sigma / d \eta_{1} d \eta_{2} d \phi_{1}\right|$ increases with $\eta$. At larger $\eta$, however, the variation of the fragmentation functions becomes important. As $\eta$ further increases, $z_{1}$ and $z_{2}$ increase and $D_{h / q}\left(z_{i}\right)$ decrease fast, leading to a decrease of $\left|\Delta d^{3} \sigma / d \eta_{1} d \eta_{2} d \phi_{1}\right|$. Such a decrease results from another reason related with the fact that we integrate 
over bins of $k_{T 1}$ and $k_{2 x}$ (Eq. (5.9)): For sufficiently large $\eta$, at $k_{T 1}^{\prime}=k_{T 1}+k_{0}$ and/or $k_{2 x}^{\prime}=k_{2 x}+k_{0}$ one has $z_{1}>1$ and/or $z_{2}>1$. Of course, in such kinematic ranges there can be no events, and in our calculation we have set $\Delta(\pi)=\Delta(K)=0$.

\section{Critical assessment and conclusions.}

Now we discuss the feasibility of the foregoing experiments and the stability and reliability of our predictions against various uncertainties. We begin with the question of how one can obtain a high energy polarized photon beam.

Polarized photons (of squared 4-momentum $q^{2} \simeq 0$ ) are produced from Bremsstrahlung (Brems) of polarized electrons deflected at very small angles. To determine the degree of photon polarization, in principle, one way is to tag the final electron (either by magnetic spectrometer or - better at high energy - by calorimetry methods) and determine the final electron's polarization by a double scattering experiment; all this is already quite hard. Moreover, in this way, the energy spectrum of the photons will have the usual Brems form, i.e. decreasing fast with photon energy $E_{\gamma}$. To produce a significant photon flux with high $E_{\gamma}$, one needs a very intense electron beam. Anyway, with degree of polarization $\sim 70 \%$ of the initial electrons one anticipates a significant degree of photon polarization.

Perhaps a better way (presently developped at SLAC and discussed elsewhere ${ }^{(24)}$ ) is via an optical laser beam. When the laser photons are backscattered on electrons, under proper arrangement, they can produce a spectrum peaked at some fairly high $E_{\gamma}$. The photons can be polarized and, in fact, with a large degree of polarization.

We turn to another question. In our predictions there are several uncertainties, and one may ask which of the observables is the most stable against (i.e. least affected by) these uncertainties. We argue that this is the asymmetry $A$, Eq. (4.2).

As we already discussed, regarding the choice of the scale $Q^{2}, A$ is more stable 
than the individual cross sections; the reason is obvious.

Uncertainty is also involved in the fragmentation functions $D_{h / c}^{(25)}$. Again $A$ is expected to be more stable since the same $D_{h / c}$ enter $E \Delta d \sigma / d^{3} p$ and $E d \sigma / d^{3} p$. Changing $D_{h / c}$ will somewhat change our predicted rates, but not by order of magnitude.

An important uncertainty concerns higher order QCD corrections (HOC). Again $A$ is expected to be more stable, and for this we argue as follows: For two different physical processes initiated by longitudinally polarized particles, namely $\vec{\gamma} \vec{p} \rightarrow \gamma$ (large- $\left.p_{T}\right)+X$ and $\vec{p} \vec{p} \rightarrow \gamma$ (large- $\left.p_{T}\right)+X$, complete next-to-leading order corrections have been determined. ${ }^{(26)(27)}$ For Drell-Yan lepton pair production by transversely polarized hadrons HOC have also been determined. ${ }^{(28)}$ In all these cases it was found that the $K$-factors are much the same as of the corresponding unpolarized processes. ${ }^{(29)(30)}$ This is not accidental: As it is discussed and analyzed in Refs. 26, 27 and 28, it is due to the fact that, to a great extent, HOC for such processes are dominated by certain terms involving distributions; and that these terms are much the same in longitudinally polarized, transversely polarized and unpolarized processes. Then we may anticipate that the $K$-factors for $\vec{\gamma} \vec{p} \rightarrow h^{ \pm} X$ and $\gamma p \rightarrow h^{ \pm} X$ will not differ much.

Another question (not unrelated to the last one) is the reliability of our predictions. The discussion on $\Delta F_{q / \gamma}$ and $\Delta F_{g / \gamma}$ (Sect. 3) shows that at $x_{T} \equiv 2 p_{T} / \sqrt{s} \sim$ 0 (physically interesting region) and at $x_{T} \sim 1$ (less interesting since cross sections are small) our predictions are not very reliable. For not too small (and not too large) $x_{T}$, however, where the effects of photon structure are unimportant, we believe that solutions 1 and 2 reliably predict the anticipated range of cross sections.

Two hadron photoproduction (Sect. 5) is a way to bypass (to a great extent) various small- $x$ uncertainties. Of course, the experiment is very difficult: In addi- 
tion to the question of the feasibility of polarized photon beams, it demands the measurement of four cross sections; and to avoid large statistical errors, high statistics are required. Also, to avoid large systematic errors, all four cross sections must be measured in the same experiment. Thus the whole Sect. 5 may seem rather academic. Nevertheless, to our knowledge, the observables $\Delta(\pi)$ and $\Delta(K)$ are among the very few (the only ?) that isolate the polarized gluon distribution, $\Delta g$. Thus we believe they deserve our Sect. 5 .

On the whole we may conclude that the processes of this paper are not to mark the beginning of determining $\Delta g$; other processes, e.g. $\vec{p} \vec{p} \rightarrow \gamma\left(\right.$ large- $\left.p_{T}\right)+X^{(27)}$, are more appropriate. But as the understanding of importance of experiments with polarized particles (as second generation tests of QCD) and the development of polarization techniques progress, these processes may serve a useful purpose.

\section{Acknowledgments}

We would like to thank D. Hanna, A. Melissinos, C. Papanikolas and P. Patel for helpful discussions. One of us (APC) thanks X. Artru, G. Bunce, A. Effremov, G. Shapiro, O. Teryaev, R. Windmolders and other participants of the $5^{\text {th }}$ International Workshop on H.E. Spin Physics ("Spin-93"), Protvino, Russia, 20-24 September 1993, as well as G. Jikia and other participants of the Joint Internat. Workshop, Zvenigorod, Russia, 15-21 September 1993, for many useful discussions. This work was also supported by the Natural Sciences and Engineering Research Council of Canada and by the Quebec Department of Education. 


\section{REFERENCES AND FOOTNOTES}

1. E. Auge et al (NA14 Collaboration), Phys. Lett. B168, 163 (1986).

2. D. Treille, Proceed. of the Internat. Europhys. Conf. on H.E. Physics, Bari, Italy, July 1985 (ed. Laterza Bari), p. 793; E. Katsoufis, Proceed. of the 2nd Internat. Hellenic School on Elem. Particle Phys., Corfu, Greece, September 1985 (World Scientific) p. 483.

3. J. Ashman et al (EM Collab.), Phys. Lett. B206, 364 (1988); Nucl. Phys. B328, 1 (1989).

4. B. Adeva et al (SMC Collaboration), Phys. Lett. B302, 533 (1993).

5. P. Anthony et al (E142 Collaboration), SLAC-PUB-6101 (1993).

6. G. Altarelli, CERN-TH.6340/91, Contribution to the DESY Workshop on Physics at HERA, Hamburg, 1991; E. Reya, Dortmund preprint DO-TH 93/09.

7. RHIC, Phenix and Star Collaborations "Proposal on Spin Physics Using the RHIC Polarized Collider", Brookhaven, August 1992; G. Bunce et al, Particle World 3, 1 (1992).

8. A. Yokosawa, Argonne report ANL-HEP-CP-90-101 and Proceed. of Polarized Collider Workshop, AIP Conference Proceed. No. 223 (1991).

9. J. Babcock, E. Monsay and D. Sivers, Phys. Rev. D19, 1483 (1979).

10. R. Baier, J. Engels and B. Petersson, Z. Phys. C2, 265 (1979).

11. R. Field and R.P. Feynman, Phys. Rev. D15, 2590 (1977).

12. A.P. Contogouris, R. Gaskell and A. Nicolaidis, Phys. Rev. D17, 839 (1978).

13. J.W. Cronin et al (Chicago-Princeton Collab.), Phys. Rev. D11, 3105 (1975) and Proceed. of the 18th Internat. Conf. on H.E. Phys., Tbilisi, 1976(JINR. Dubna), Vol. I, p. A4-23; M. Albrow et al (British-French-Scandinavian Collab.). Nucl. Phys. B135. 461 (1978) and B145, 305 (1978). 
14. G. Altarelli and G. Parisi, Nucl. Phys. B126, 298 (1977).

15. Ch. Berger and W. Wagner, Phys. Rep. 146, 1 (1987); see also H. Abramowicz et al, Int. J. Mod. Phys. A8, 1005 (1993).

16. E. Witten, Nucl. Phys. B120, 189 (1977).

17. I. Antoniadis and G. Grundberg, Nucl. Phys. B213, 445 (1983); I. Antoniadis and L. Marleau, Phys. Lett. B161, 163 (1985).

18. M. Glück and E. Reya, Phys. Rev. D28, 2743 (1983); M. Drees et al, Z. Phys. C27, 587 (1985).

19. J. Hassan and D. Pilling, Nucl. Phys. B187, 563 (1981).

20. As a check, we have determined the photon structure contribution to $E \Delta d \sigma / d^{3} p$ from $\gamma \rightarrow u \bar{u}$ and $\vec{g} \vec{u} \rightarrow g u$ using $\Delta F_{u / \gamma}$ of Ref. 19, and compared it with ours. In general, the difference is very small, even at small $p_{T}$.

21. G. Altarelli and W. Stirling, Particle World 1, 40 (1989).

22. J. Morfin and W.-K. Tung, Z. Phys. C52, 13 (1991).

23. M. Fontannaz et al, Z. Phys. C6, 357 and 241 (1980).

24. There has been much discussion of a future high energy $\gamma-\gamma$ accelerator in Russia with Western participation; see Proceedings of the Joint International Workshop, Zvenigorod, Russia, 15-21 September 1993. Unpolarized and polarized (with significant degree of polarization) photon beams are considered.

25. Our fragmentation functions ${ }^{(10)}$ are outdated. Well after the completion of our calculations, we became aware of a very recent determination, P. Chiappetta et al, CPT-92-PE-2841. With them our results may somewhat change, but not by order of magnitude.

26. A.P. Contogouris, S. Papadopoulos, and F. Tkachov, Phys. Rev. D46, 2846 (1992).

27. A.P. Contogouris, B. Kamal, Z. Merebashvili, and F. Tkachov: Phys. Lett. 
B304, 329 (1993); Phys. Rev. D48, to appear in the issue of 1 November 1993.

28. A.P. Contogouris, B. Kamal, and Z. Merebashvili, Proceed. of Joint Internat. Workshop, Zvenigorod, Russia, 15-21 Sept. 1993 (to be published in Yadernaya Fizika).

29. The same holds for Drell-Yan lepton pair production in longitudinally polarized hadron collisions. ${ }^{(30)}$ This, however, follows trivially by the fact that emission of a (massless or massive) vector meson does not flip the helicity of a massless quark (helicity conservation); as a result, both Born term and 1-loop HOC for longitud. polarized Drell-Yan follow from the corresp. unpolarized by a simple change of sign. Note that, in general, with 1-loop HOC, parton distributions and fragmentation functions evolved via 2-loop anomalous dimensions and running coupling to 2-loop order, the sensitivity in e.g. the scale $Q^{2}$ is significantly reduced.

30. P. Ratcliffe, Nucl. Phys. B233, 45 (1983). 


\section{FIGURE CAPTIONS}

Fig. 1. Inclusive cross sections for $\vec{\gamma} \vec{p} \rightarrow \pi^{ \pm}+X$ at $\sqrt{s}=27$ and $170 \mathrm{GeV}$. Long dashed lines: Direct subprocess contributions._ Short dashed: Photon structure contributions._Solid: total contribution: (a) At rapidity $\eta=1.5$._ (b) At $\eta=0$. use of logarithmic scale, we have multiplied the indicated cross section by -1 .

Fig. 2. The ratio of the total inclusive cross section for $\vec{\gamma} \vec{p} \rightarrow \pi^{ \pm}+X$ determined with solution 1 to that determined with solution 2 of Ref. 16 at $\eta=0,-1.5$ and 1.5 .

Fig. 3. Inclusive cross sections for $\vec{\gamma} \vec{p} \rightarrow h^{ \pm}+X$, where $h=\pi$ or $K$, integrated over bins of $p_{T}$.

Fig. 4. The asymmetries $A\left(p_{T}, s, \eta\right)$, Eq. (4.2).

Fig. 5. Kinematics of the reaction $\gamma p \rightarrow h_{1}+h_{2}+X$ with the hadrons $h_{1}, h_{2}$ produced in opposite azimuthal hemispheres.

Fig. 6. The combinations $\Delta(\pi)+\Delta(K)$ (Sect. 5) of inclusive cross sections for $\vec{\gamma} \vec{p} \rightarrow$ $h_{1}+h_{2}+X$ integrated over bins of $k_{T 1}^{\prime}$ and $k_{2 x}^{\prime}$ (Eq. (5.9)). 
$\sqrt{s}=27^{\circ} \mathrm{GeV}, \quad \eta=1.5$
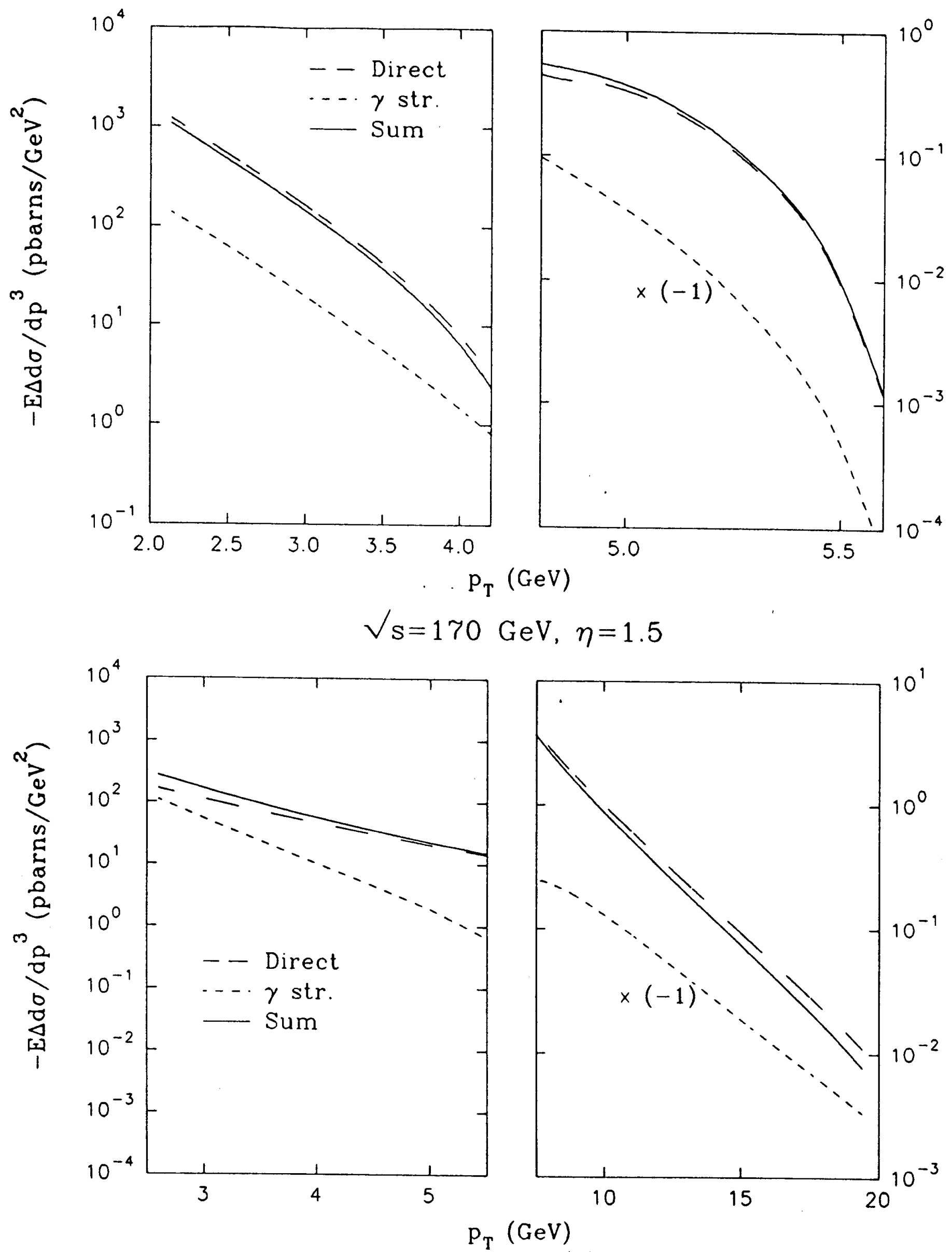

Figure 1(a) 
$V \mathrm{~s}=27 \mathrm{GeV}, \eta=0$
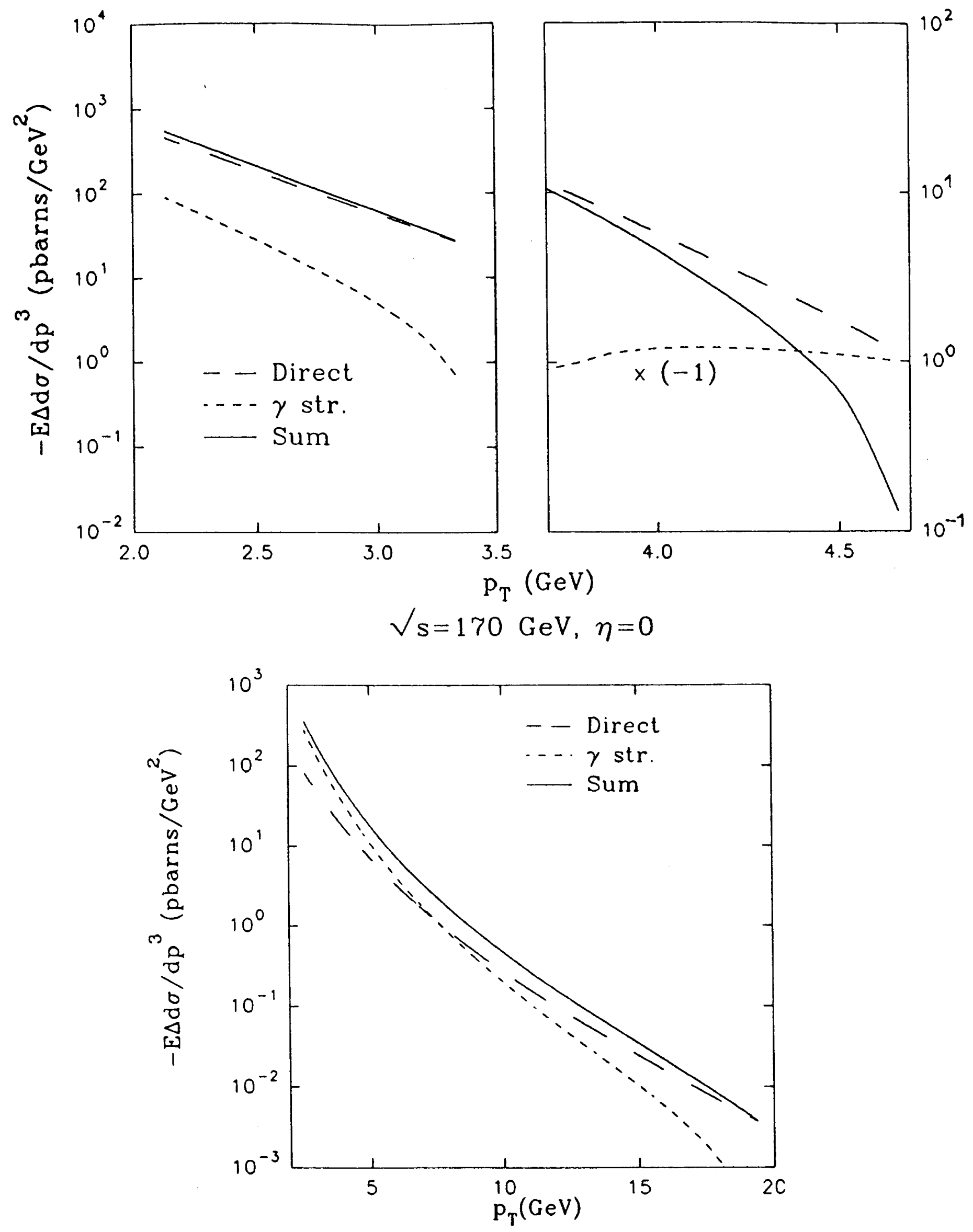

Figure 1(b) 
$V_{\mathrm{s}}=27 \mathrm{GeV}, \eta=-1.5$
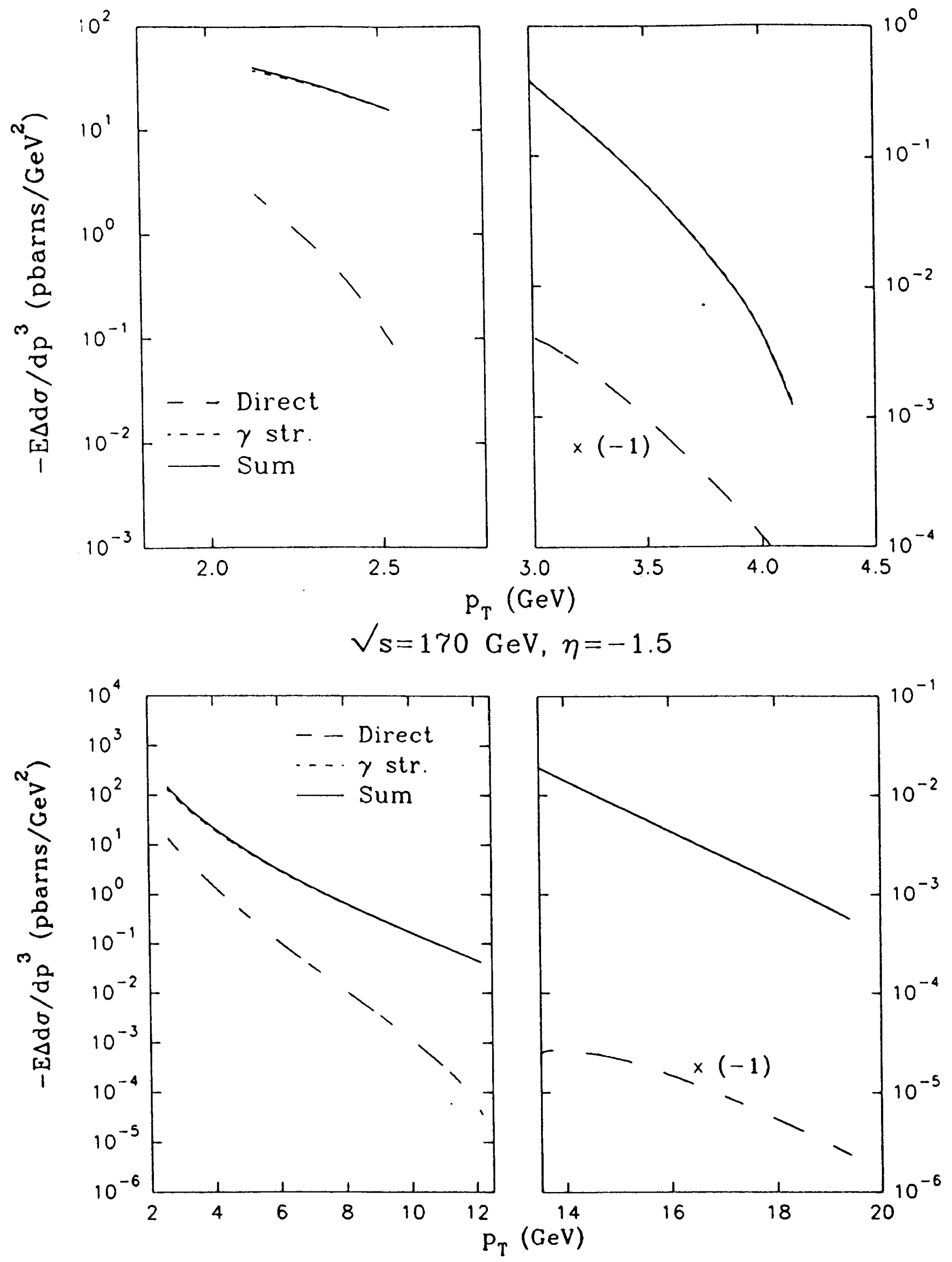

Figure 1(c) 

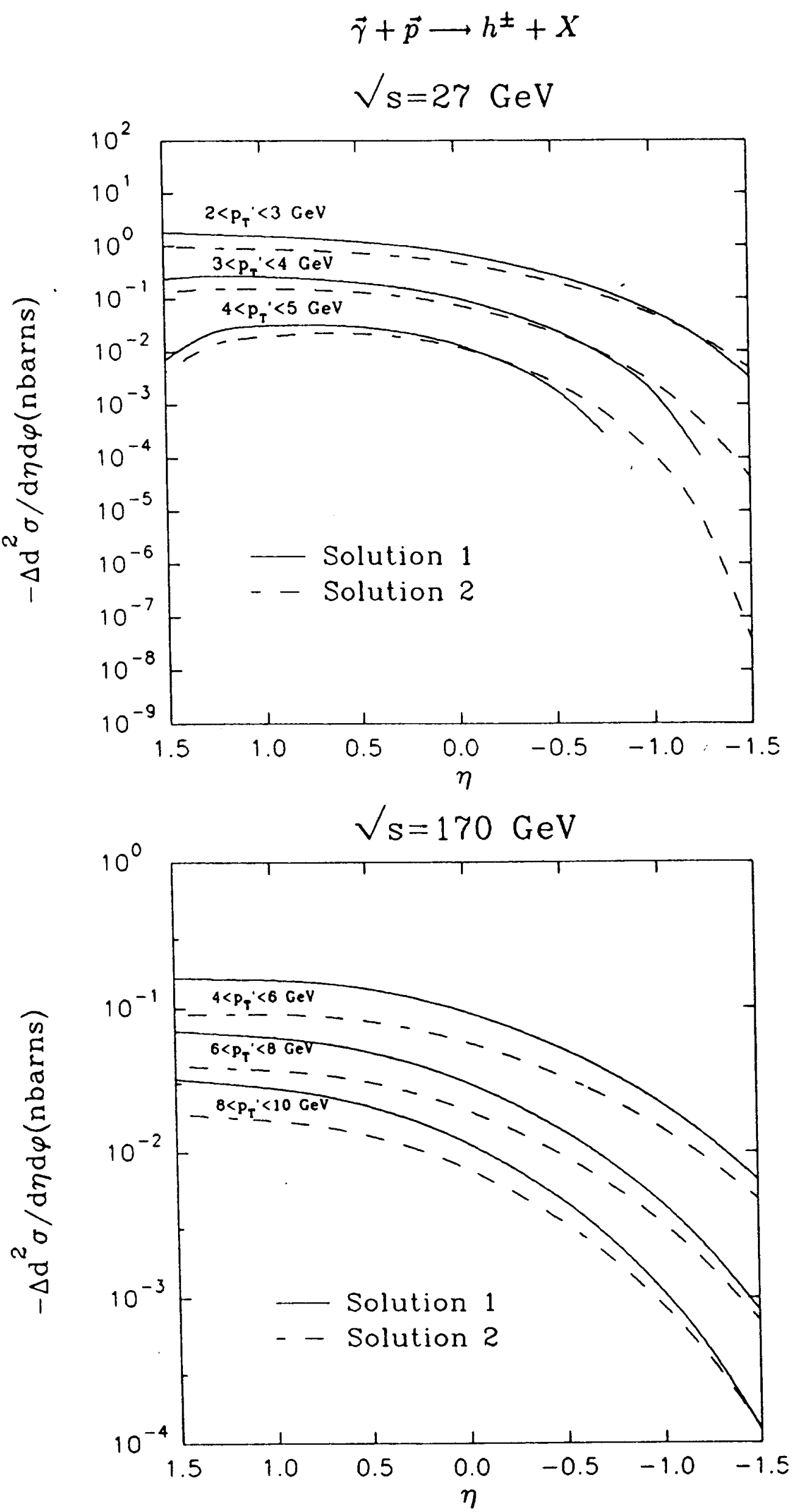

Figure 3 

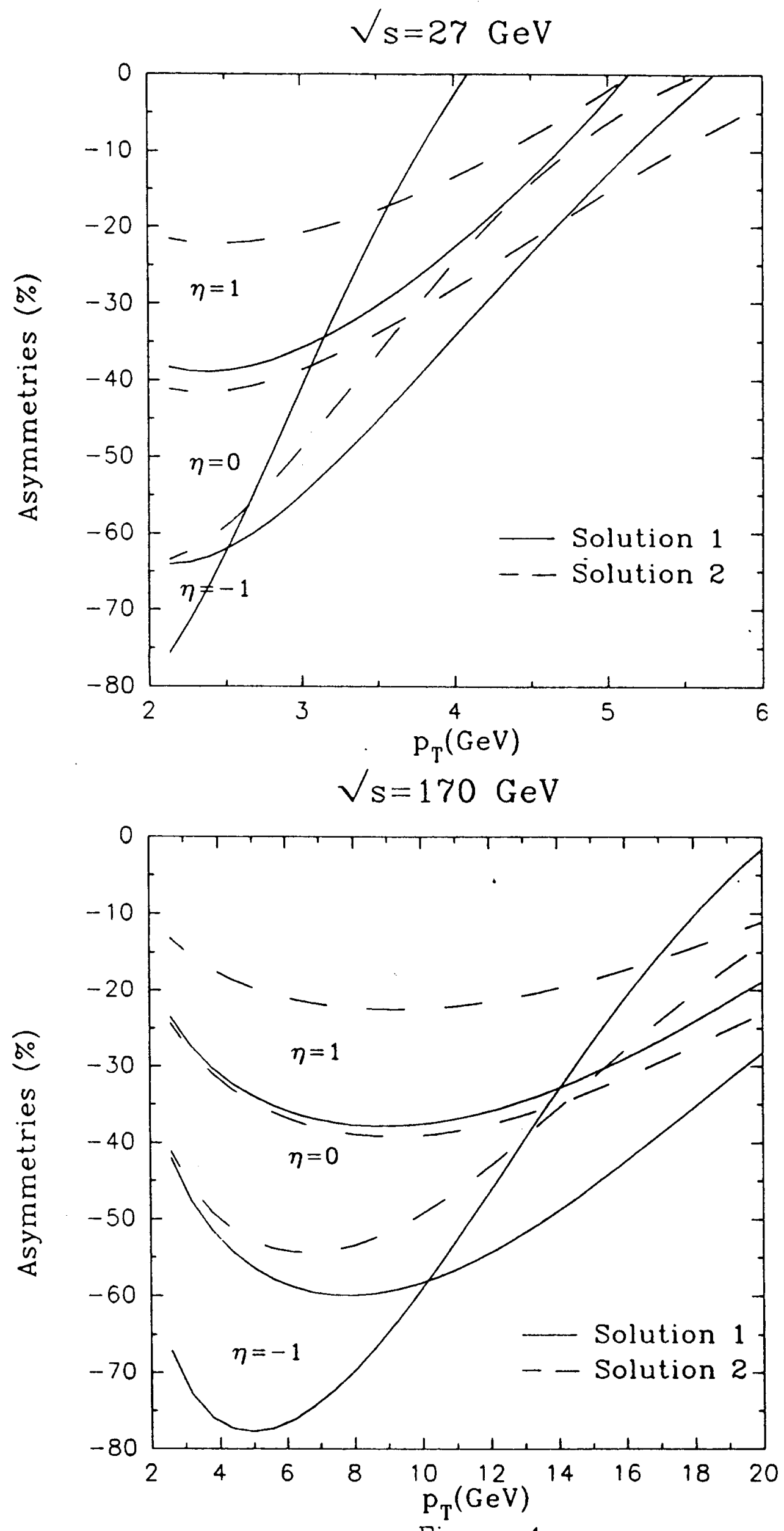

Figure 4 


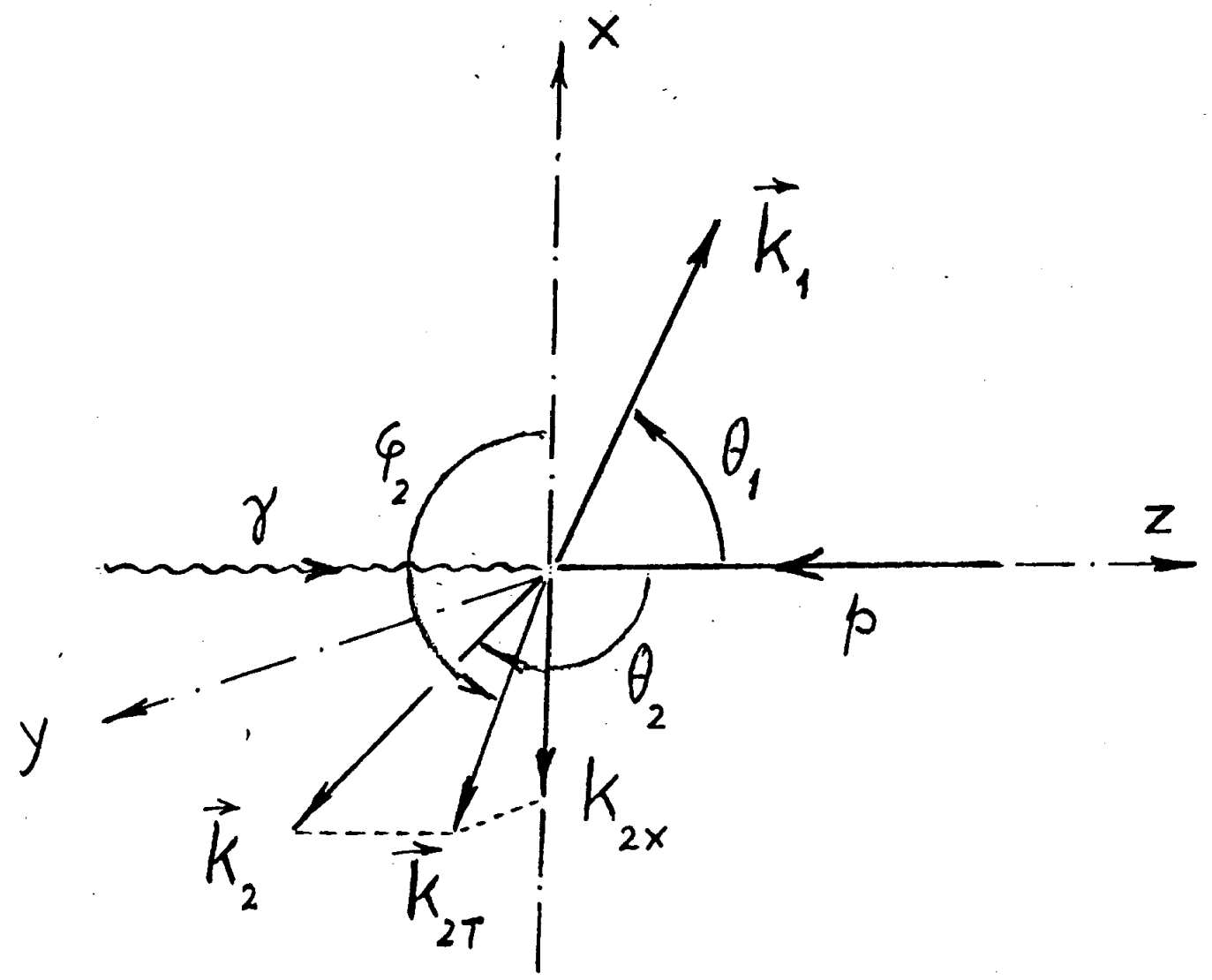

Figure 5 

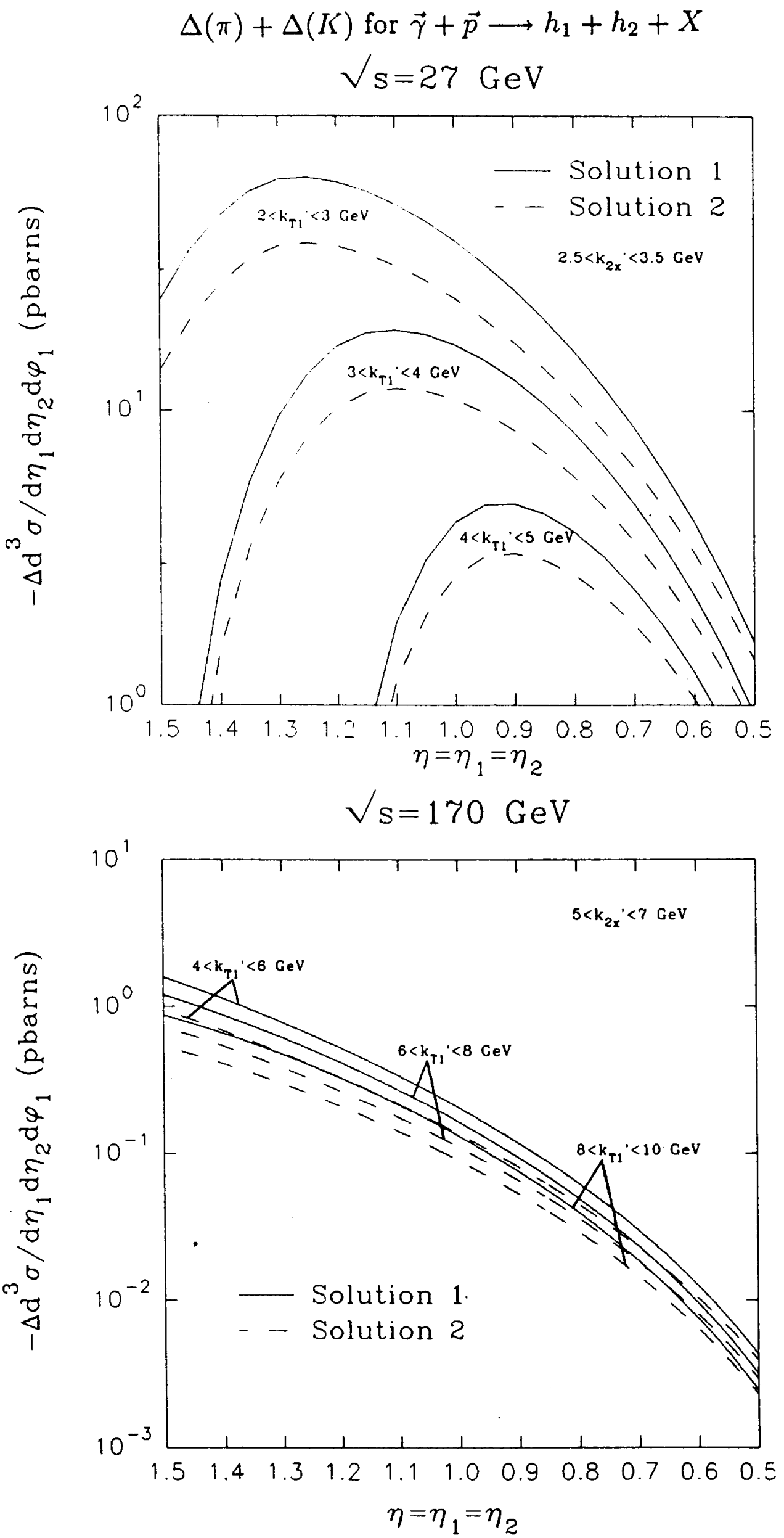

Figure 6 